\title{
Bacterial microcosms obey Taylor's law: effects of abiotic and biotic stress and genetics on mean and variance of population density
}

\author{
Oliver Kaltz ${ }^{1}$ Patricia Escobar-Páramo ${ }^{1,2}$, Michael E Hochberg ${ }^{1}$ and Joel E Cohen ${ }^{3^{*}}$
}

\begin{abstract}
Introduction: According to the empirical regularity called Taylor's law, the variance of population density in samples of populations is a power of the mean population density. The exponent is often between 1 and 2. Our experiments investigated how genetics, evolution, and environment shape Taylor's law.
\end{abstract}

Methods: Genetically different strains (wild type and hypermutator) of the bacterium Pseudomonas fluorescens evolved and were assayed under different environmental conditions (with and without antibiotic rifampicin and bacteriophage SBW25 $\phi 2$, separately and in combination).

Results: Experimental treatments altered the exponent $b$, but not the power law form, of the relation between variance and mean population density. Bacterial populations treated only with rifampicin had a narrow range of mean population densities and exponent $b=5.43$. Populations exposed to rifampicin plus phage had $b=1.51$. In ancestral, control, and phage-exposed populations, mean abundance varied widely and $b$ was not significantly different from 2. Evolutionary factors (mutation rate, selection) and ecological factors (abiotic, biotic) jointly influenced $b$.

Conclusions: Taylor's power law relationship accurately and robustly described variance as a function of mean population density, with overall exponent $b=1.89$. These and other experiments with different factors acting on bacterial population size support the relevance of models that predict 'universal' patterns of fluctuation scaling.

Keywords: Taylor's law, Pseudomonas fluorescens, rifampicin, bacteriophage, genetics

\section{Introduction}

In 1961, L. R. Taylor brought to wide attention an approximate empirical power law relationship, variance $=a(\text { mean })^{b}$, where $a>0$ and $b>0$, between the variance and mean of the size of insect populations (Taylor 1961). Taylor's law describes variation in hundreds of species and has been the subject of approximately a thousand papers (Eisler et al. 2008). Taylor's law has practical importance for designing efficient sampling of agricultural pests and insect vectors of human diseases (Young and Young 1994; Binns et al. 2000; Park and Cho 2004).

\footnotetext{
* Correspondence: cohen@rockefeller.edu

${ }^{3}$ Laboratory of Populations, Rockefeller and Columbia Universities, 1230 York Avenue, Box 20, New York, NY, 10065-6399, USA

Full list of author information is available at the end of the article
}

Long before Taylor publicized this empirical pattern, Luria and Delbrück (1943) investigated, theoretically and experimentally, mutations of bacteria from virus sensitivity to virus resistance. If virus resistance arose from heritable mutations, then, their theory showed, the distribution of the number of resistant bacteria under given conditions would not be described by the Poisson distribution, which has variance equal to mean, but would be described by an over-dispersed distribution, with variance significantly larger than the mean. They counted the numbers of resistant bacteria and reported the raw counts, means, and variances in series of eight similar cultures in their Table 2. They remarked (Luria and Delbrück 1943, page 504): '... in every experiment the fluctuation of the numbers of resistant bacteria is tremendously higher than could be accounted for by the sampling errors, ... in conflict with the expectations 
from the hypothesis of acquired immunity' and in support of the alternative hypothesis of heritable mutations.

They did not remark that the relation between the variance and the mean in their data is well approximated by a power law. Showing that a power law applies to relationships between the mean and variance of population size suggests orderly underlying processes (Taylor et al. 1983, but see, e.g., Hanski 1982; Anderson et al. 1982). We performed a linear regression of the $\log _{10}$ variance as a function of the $\log _{10}$ mean in the data of Table 2 of Luria and Delbrück (1943) and found that $b=1.47 \pm 0.57$ (standard error) with $R^{2}=0.53$. This estimate of $b$ differed from 0 significantly (95\% level) but did not differ significantly from either 1 or 2 .

The present paper uses Taylor's law to extend the research of Luria and Delbrück (1943) beyond genetics into ecology and evolution. We ask here how genetic, ecological, and evolutionary processes interact to affect variance-mean relationships. Are specific conditions necessary to generate Taylor's law, or is Taylor's law robust with respect to other ecological and evolutionary processes?

Models developed in other areas of population biology suggest that Taylor's law may be quite robust. For example, in models of stem cell clones, stochastic 'voter' or 'random walk' models of neutral drift converge to 'universal' patterns which obey Taylor's law with $b=2$ for 'any mechanism of stem cell self-renewal, however complex' (Klein and Simons 2011, page 3107). If these patterns are universal, then they should apply to bacterial populations. In a Lotka-Volterra model of interspecific competition, under a range of conditions, Taylor's law holds with $1 \leq b$ $\leq 2$ (Kilpatrick and Ives 2003). These theoretical suggestions need to be tested experimentally.

We used microbial microcosms under controlled initial conditions and in a controlled environment to investigate the impacts of genetic differences in mutation rate, exposures to an abiotic stressor (antibiotic), and exposures to a biotic stressor (parasitism by a lytic bacteriophage) on a single bacterial species' adherence to Taylor's law over an evolutionary time scale (approximately 50 generations). Ramsayer et al. (2011) used the same species of bacterium to investigate the impacts of competition from another bacterial species and of nutrient concentrations on the adherence to Taylor's law of each competitor, and measured impacts on an ecological time scale $(24 \mathrm{~h})$ of, at most, a few generations. The shared purpose of both studies is to identify the ecological and evolutionary factors that contribute to the success or failure of Taylor's law and that influence the values of its parameters.

\section{Methods}

The gram-negative bacterium Pseudomonas fluorescens SBW25 has been employed to study adaptation in heterogeneous environments (Venail et al. 2008; Buckling et al. 2009) and coevolution with a lytic bacteriophage SBW25 $\phi 2$ (Brockhurst et al. 2007; Poullain et al. 2008). We analyzed in a new way the data of EscobarPáramo et al. (2012) on the population dynamics and evolution of four strains of $P$. fluorescens SBW25 exposed to a biotic and an abiotic stress. The four strains, derived from the wild-type (WT) (Rainey and Bailey 1996), included an isogenic hypermutator (MutS) knock-out mutant with a per base mutation rate of approximately $10^{-5}$ per generation (Pal et al. 2007) (compared to approximately $5 \times 10^{-7}$ per base pair per generation in $W T$ ), and lines resistant to the antibiotic rifampicin $(R i f+)$, one derived from the $W T$ and the other from the MutS strain. Thus, the experimental replicate populations of $P$. fluorescens were seeded from four different strains of bacterial cells: wild type WT/Rif(Rainey and Bailey 1996), hypermutator MutS/Rif- (Pal et al. 2007), rifampicin-resistant wild type WT/Rif+, and rifampicin-resistant hypermutator $M u t S / R i f+$. Over approximately 50 generations, 12 replicate populations of each strain were submitted (or not submitted, in the control) to antibiotic rifampicin (rif), an abiotic stressor, and to the lytic bacteriophage SBW $25 \phi 2$, a biotic stressor, separately and in combination, for a total of four treatments. At the end of the experiment, densities (number of colony-forming units per milliliter) of evolved populations were assayed on King's B (KB) agar plates in the presence $(\mathrm{KB}+\mathrm{rif})$ or absence $(\mathrm{KB})$ of rif to distinguish total count from the antibiotic-resistant subpopulation. Escobar-Páramo et al. (2012) give further details of experimental methods.

We calculated means and variances of final bacterial density across the replicate populations of each combination of bacterial mutation rate, experimental treatment, and assay plate environment. The table in Additional file 1 gives the resulting 40 pairs of means and variances. As means and variances were calculated over distinct replicate populations at a given time, and not for individual replicates observed over time, we examined whether these final means and variances and those of the ancestral lines obeyed the ensemble (or equivalently, spatial) form of Taylor's law, and if so, whether $b$ of Taylor's law depended on the ancestral lines, experimental treatments, or assay plate environment (SAS 2009; Mathworks 2011).

\section{Results}

Mean bacterial densities covered nearly 8 orders of magnitude (Figure 1a). On assay plates with rif, mean population density varied from nearly $10^{2}$ to nearly $10^{10}$. The high densities arose from Rif+ (rif-resistant) clones. In the assay plates without rif, mean population density was always $>10^{8}$ [see table in Additional file 1]. 


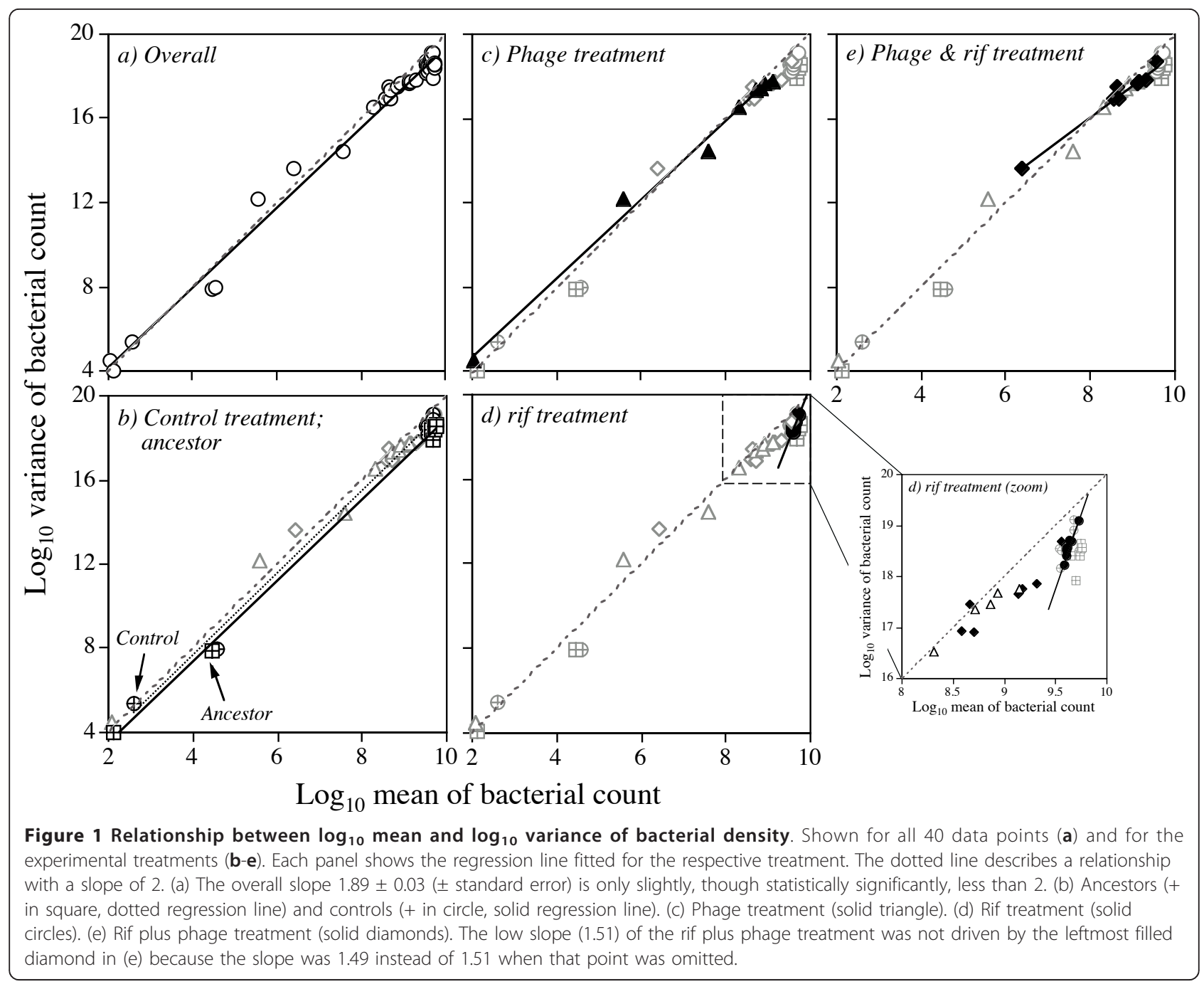

For all 40 data points combined, log variance was highly significantly positively related to log mean of bacterial density $\left(F_{1,38}=692, p<0.0001\right)$. We refer to the power $b$ in Taylor's law as the slope when $b$ is estimated from log variance $=\log a+b \log$ mean by linear regression. The point estimate $b=1.89$ fell statistically significantly below 2 (Table 1 ). There was no significant evidence of quadratic nonlinearity (the quadratic term in polynomial regression: $F_{1,37}=3.58, p=0.0663$ ).

In the minimal adequate analysis of covariance (ANCOVA) model (Table 2), the only statistically significant effects on log variance were due to log mean, to treatment, and to treatment $\times \log$ mean interaction, indicating differences in slope among treatments. Bacterial populations treated only with rif showed a very high slope (5.43), whereas populations exposed to rif plus phage showed a relatively low slope (1.51). Both slopes differed significantly from 2 (Table 1 ).
In contrast, ancestral and control populations and phage-exposed populations had slopes not significantly different from 2, whether assayed in the $\mathrm{KB}$ agar plates with or without rif. Any slope within the intersection of the confidence intervals of these three treatments, namely $(1.84,2.01)$, was compatible with the data from all three treatments. The confidence interval of the pooled data from all treatments, namely $(1.83,1.96)$, fell almost entirely within the intersection of the confidence intervals of these three treatments (Table 1). The exclusion of slope 2 by the confidence interval of the pooled data evidently derives from the two treatments with rif, with or without phage. Bootstrap analyses [see Additional file 2] confirmed these conclusions.

The rif (without phage) treatment had the slope most different from 2 and was the only one where the confidence interval lay above 2 . In this treatment, $\log _{10}$ mean ranged from 9.6 to 9.7 . This very limited range gives 
Table 1 Estimates of intercept and slope ( \pm standard error) from linear regressions of log-variance on log-mean

\begin{tabular}{|c|c|c|c|c|c|}
\hline & Intercept & Slope & $\begin{array}{c}95 \% \text { C.I. } \\
\text { [lower; upper] }\end{array}$ & $\begin{array}{c}\text { Adjusted } \\
R^{2}\end{array}$ & $n$ \\
\hline Overall & $0.38 \pm 0.29$ & $1.89 \pm 0.03$ & {$[1.83 ; 1.96]$} & 0.99 & 40 \\
\hline \multicolumn{6}{|l|}{ Plate } \\
\hline KB & $5.62 \pm 1.23$ & $1.33 \pm 0.13$ & {$[1.06 ; 1.61]$} & 0.85 & 20 \\
\hline$K B+$ rif & $0.28 \pm 0.36$ & $1.91 \pm 0.04$ & {$[1.81 ; 2.00]$} & 0.99 & 20 \\
\hline \multicolumn{6}{|l|}{ Strain } \\
\hline MutS/Rif- & $-0.36 \pm 0.83$ & $1.97 \pm 0.10$ & {$[1.75 ; 2.19]$} & 0.98 & 10 \\
\hline MutS/Rift & $3.47 \pm 1.78$ & $1.57 \pm 0.19$ & {$[1.14 ; 2.00]$} & 0.89 & 10 \\
\hline WT/Rif- & $0.44 \pm 0.25$ & $1.87 \pm 0.03$ & {$[1.80 ; 1.94]$} & 0.99 & 10 \\
\hline WT/Rift+ & $2.94 \pm 0.98$ & $1.62 \pm 0.11$ & {$[1.37 ; 1.87]$} & 0.96 & 10 \\
\hline Rif- (pooled) & $0.22 \pm 0.34$ & $1.90 \pm 0.04$ & {$[1.82 ; 1.99]$} & 0.99 & 20 \\
\hline Rif + (pooled $)$ & $2.98 \pm 0.75$ & $1.62 \pm 0.08$ & {$[1.45 ; 1.79]$} & 0.96 & 20 \\
\hline WT (pooled) & $0.62 \pm 0.31$ & $1.87 \pm 0.04$ & {$[1.79 ; 1.94]$} & 0.99 & 20 \\
\hline MutS (pooled) & $-0.25 \pm 0.61$ & $1.96 \pm 0.07$ & {$[1.82 ; 2.11]$} & 0.98 & 20 \\
\hline \multicolumn{6}{|l|}{ Treatment } \\
\hline Ancestor & $-0.30 \pm 0.31$ & $1.92 \pm 0.04$ & {$[1.84 ; 2.01]$} & 0.99 & 8 \\
\hline Control & $-0.21 \pm 0.52$ & $1.95 \pm 0.06$ & {$[1.81 ; 2.10]$} & 0.99 & 8 \\
\hline Rifampicin & $-33.71 \pm 7.12$ & $5.43 \pm 0.74$ & {$[3.68 ; 7.18]$} & 0.90 & 8 \\
\hline Phage & $0.97 \pm 0.56$ & $1.86 \pm 0.07$ & {$[1.69 ; 2.03]$} & 0.99 & 8 \\
\hline Rifampicin and phage & $3.95 \pm 0.81$ & $1.51 \pm 0.09$ & {$[1.29 ; 1.73]$} & 0.97 & 8 \\
\hline
\end{tabular}

Least-squares regressions of $\log _{10}$ variance of bacterial density on $\log _{10}$ mean density (in colony-forming units per milliliter) were fitted across all data (overall) or fitted separately for the different assay environments (plate), bacterial strains, and long-term treatments. For each regression, the $95 \%$ confidence interval (C.I.) for the slope is given. Bold type indicates slopes significantly different from +2 (upper and lower boundaries calculated as mean slope $\pm t_{n-1,95 \%} \times$ standard error).

False discovery rate method was used to correct for multiple testing (García 2004). Also given are the adjusted $R^{2}$ and the number of data points ( $n$ ).

little information about how log variance would vary over multiple orders of magnitude of changes in log mean.

Rif plus phage was the other treatment with slope significantly different from 2 . In this treatment, $b<2$ and log mean ranged from 6.4 to 9.6. This range of log mean is more informative than the very narrow range of the log mean in the rif treatment, but is notably less than that of the other three treatments (ancestor, control, phage only) where log mean varied over at least 6 orders of magnitude (from $<3$ to $>9$ ). The range of mean population densities and the slope of the rif plus phage treatment resembled those of the phage (alone) treatment far more than those of the rif (alone)

Table 2 Analysis of covariance after sequential removal of nonsignificant $(p>0.1$ ) factors from the full model

\begin{tabular}{lcccc}
\hline Source & $\boldsymbol{d f}$ & Mean squares & $\boldsymbol{F}$ & $\boldsymbol{P}$ \\
\hline Log mean & 1 & 1.555 & 14.30 & 0.0008 \\
Treatment & 4 & 0.707 & 6.49 & 0.0008 \\
$($ Rift, Rif-) strain & 1 & 0.148 & 1.36 & 0.2533 \\
Treatment $\times \log$ mean & 4 & 1.390 & 3.19 & 0.0280 \\
$($ Rift, Rif-) strain $\times \log$ mean & 1 & 0.408 & 3.75 & 0.0630 \\
Residual & 28 & 0.109 & & \\
\hline
\end{tabular}

Analysis of covariance of the $\log _{10}$ variance of bacterial density, as a function of the $\log _{10}$ mean density, treatment (rifampicin, phage, rifampicin plus phage, control, ancestor), and bacterial resistance strain (Rift, Rif-), in a minimal model. treatment. Further, in the rif plus phage treatment, initially rif-resistant lines had the lowest means, whereas in the rif (alone) treatment, initially rif-resistant lines tended to have the highest means. The difference is evidently due, in part at least, to the action of the phage on the bacteria.

\section{Discussion}

A linear relation between log variance and log mean (Taylor's law) described accurately and robustly how variability in population size changed with mean population size in P. fluorescens bacteria. Though strains with different mutator genotypes and antibiotic-resistant phenotypes evolved and were assayed under different environmental conditions, no factors in our experiments significantly altered the linearity of the relationship.

In a combined analysis of covariance when all cofactors were considered simultaneously, the experimental treatments were the only factors that affected the slope $b$ of Taylor's law (Table 2). In this integrated analysis, neither the bacterial strain nor the assay plate significantly affected $b$. Among the treatments, the slope was indistinguishable for ancestors, controls, and populations treated with phage only.

The exceptionally high slope of the rif treatment and the exceptionally low slope of the treatment with rif plus phage require further confirmation by experiments that are designed to produce a wider range of variation 
in log mean. Such variation in log mean density might be achieved by varying the concentrations of rif, phage, and KB nutrients. Ramsayer et al. (2011) showed that variation in $\mathrm{KB}$ concentration produced wide variation in mean bacterial densities and that Taylor's law applied. Our study extends and generalizes these findings on Taylor's law to the effects of abiotic and biotic environments, and evolutionary potential in the form of mutation rates.

Mean population sizes ranged much more widely for some genetic strains and experimental treatments than for others, but whether these differences in mean affected the variance within sets of replicates, that is, the mean-variance relationship, was not clear without further investigation.

When different strains and treatments were analyzed separately (Table 1), rather than as parts of a single experiment, estimates of slope differed by strain and treatment, though no strain or treatments rejected the power law form of Taylor's law. In particular (strain section in Table 1), WT strains had slopes significantly less than 2, while MutS strains had slopes not significantly different from 2. Hence, for a given increase in mean population size, the variances of $W T$ populations increased by a smaller amount than the variances of MutS populations. In this instance, the lower genetic diversity of WT compared to MutS is associated with a lower rate of increase in the variance of population size. This possible association of genetic strain with slope suggested that the mutation rate of the strain (as a source of genetic variation) played a role in determining the slope of the mean-variance relationship.

The speculative inferences of the previous paragraph should be viewed as hypotheses for verification in future experiments rather than as statistically well-grounded conclusions, for two reasons. First, the confidence intervals of the slopes of WT strains overlapped greatly with the confidence intervals of the slopes of the corresponding MutS strains (Table 1). Second, the difference between strains attained statistical significance here because this analysis pooled all the different treatments which WT strains experienced and pooled all the different treatments which MutS strains experienced, thereby increasing the sample size of each to attain apparent statistical significance. The earlier analysis of the experiment as a whole took account of the factorial design of the experiment and distinguished the populations of each strain that received each treatment; that analysis revealed no significant effect of strain on slope.

In addition to the possible genetic association with slope, MutS/Rif- populations had higher slope than MutS/Rif+ populations and, likewise, WT/Rif- populations had higher slope than WT/Rif+ populations (again, with overlapping confidence intervals in each comparison). Together, these results suggested (weakly) that a combination of mutation and selection determined the values of $b$ : hypothetically, MutS may have increased $b$ over the course of the experiment by increasing the variance in growth rates as a result of increased rates of mutation, whereas the lower slope of the Rif+ populations reflects the action of selection in the past. This suggestion merits further experimental and field testing. Do some natural populations have low slope because of the action of selection on them?

If the rif treatment affects the slope in future experiments where log mean density varies more widely, then we could interpret the effect as follows: Culturing bacteria with rif selected for genes that conferred rif resistance, driving a divergence in population growth rates among populations in which different resistant alleles (demonstrated at the molecular level by Escobar-Páramo et al. 2012, their Table 2) arose by different mutations. Populations cultured with rif always gave rise to one or more resistant alleles and did not go extinct. The growth rate of populations derived from different mutants that arose among the bacteria cultured with rif was significantly different, according to a one-way analysis of variance (not shown here). This putative varianceincreasing effect of rif-driven selection did not seem to operate in the simultaneous presence of phage, because in the rif plus phage treatment, the slope was lower, rather than higher, than 2 . The molecular sequence data of Escobar-Páramo et al. (2012, their Table 2) may explain why the slope was significantly below 2 in the rif plus phage treatment: in this treatment, all $M u t S$ strains converged to a single allele, while in $W T$, the phage went extinct in half of the populations and the others reverted to the rif-resistant phenotype and the resulting strains were all biofilm producers. These observations suggest an interaction between abiotic (rif) and biotic (phage) selective pressures on the bacterial populations (see further discussion in Escobar-Páramo et al. 2012). Indeed, coevolving phage has been shown to affect adaptive radiation of $P$. fluorescens under variable environmental conditions (Benmayor et al. 2008).

An alternative explanation (suggested by a reviewer) of why all slopes were significantly greater than 1 (which is the slope given by Poisson distributions) could be that sampling error increased with mean abundance. For example, if some cells clumped at high density, variance among high-density cultures could increase spuriously. Unfortunately, we cannot test this idea in our data, because we had no replicate counts per plate for a given population on a given plate type. However, a clear advantage of using microcosms to study Taylor's law is that sampling error can be rejected empirically in future experiments, for example, by plotting variance as a 
function of sampling effort for different values of mean abundance.

\section{Conclusions}

The near approximation to $b=2$ (Figure 1a) and the simplicity of the ANCOVA results (Table 2), plus the excellent fit to Taylor's law with $b=2$ in the experiments of Ramsayer et al. (2011), despite completely different sets of factors acting on population size in those experiments and these, support the relevance to these experiments of the 'universal' patterns of scaling in models and data of stem cell populations (Klein and Simons 2011). Examining microbial microcosms in the light of Taylor's law is a potentially powerful tool for investigating mechanisms and consequences of ecological and evolutionary interactions. Future theoretical and experimental studies should test the generality of our findings for the same and other taxa amenable to experimental evolution.

\section{Additional material}

Additional file 1: Table of means and variances. This table gives $\log _{10}$ mean and $\log _{10}$ variance by assay plate, bacterial origin, resistance status, treatment, and number of replicates.

Additional file 2: Data analysis, including bootstrapping and results This Additional file gives details of data analysis, including bootstrapping, and the results of the bootstrap analyses.

\section{Acknowledgements}

The collection of the data analyzed here was funded by grants from the French Agence National de la Recherche (ANR) 'CoMute' (ANR-06-BLAN0164) and 'EvolStress' (ANR-09-BLAN-099-01). MEH and OK were supported by grants 'EvolStress' (ANR-09-BLAN-099-01) and 'EvoRange' (ANR-09-PEXT011). JEC was supported in part by grant EF-1038337 from the U.S. National Science Foundation, a grant from the region of Languedoc-Roussillon through the University of Montpellier 2, the assistance of Priscilla K. Rogerson, and the hospitality of Michael E. Hochberg and family during this work. Two referees provided helpful criticisms and suggestions. This is publication ISE-M 2012-032.

\section{Author details}

IInstitut des Sciences de l'Evolution, UMR CNRS-UM2-IRD, 5554, CC 065, Université Montpellier 2, Place Eugène Bataillon, Montpellier Cedex 05, 34095, France ${ }^{2}$ Centro Internacional de Física (CIF); Biotechnology Group, Universidad Nacional de Colombia, Cra 30 № 45-03, Bogotá, DC, Colombia ${ }^{3}$ Laboratory of Populations, Rockefeller and Columbia Universities, 1230 York Avenue, Box 20, New York, NY, 10065-6399, USA

\section{Authors' contributions}

OK conducted the statistical analysis, prepared the figure, and helped write the paper. PEP helped design, and conducted, the laboratory experiments, contributed to interpreting the results, and helped write the paper. MEH helped design the laboratory experiments, suggested their relevance to Taylor's law, and helped write the paper. JEC carried out the preliminary statistical analyses, performed the bootstrap analyses, and was responsible for integrating and revising the manuscript. All authors read and approved the final manuscript.
Received: 12 January 2012 Accepted: 3 April 2012

Published: 3 April 2012

\section{References}

Anderson RM, Gordon DM, Crawley MJ, Hassell MP (1982) Variability in the abundance of animal and plant species. Nature 296:245-248. doi:10.1038/ $296245 \mathrm{a} 0$.

Benmayor R, Buckling A, Bonsall MB, Brockhurst MA, Hodgson DJ (2008) The interactive effects of parasites, disturbance, and productivity on experimental adaptive radiations. Evolution 62:467-477. doi:10.1111/j.1558-5646.2007.00268. $x$.

Binns MR, Nyrop JP, van der Werf W (2000) Sampling and monitoring in crop protection: the theoretical basis for developing practical decision guides. $\mathrm{CABI}$, Oxon

Brockhurst MA, Morgan AD, Fenton A, Buckling A (2007) Experimental coevolution with bacteria and phage: the Pseudomonas fluorescens-Phi2 model system. Infect Genet Evol 7:547-552. doi:10.1016/..meegid.2007.01.005.

Buckling A, Craig Maclean R, Brockhurst MA, Colegrave N (2009) The Beagle in a bottle. Nature 457:824-829. doi:10.1038/nature07892.

Eisler Z, Bartos I, Kertész J (2008) Fluctuation scaling in complex systems: Taylor's law and beyond. Adv Phys 57:89-142. doi:10.1080/00018730801893043.

Escobar-Páramo P, Gougat-Barbera C, Hochberg ME (2012) Evolutionary dynamics of separate and combined exposure of Pseudomonas fluorescens SBW25 to antibiotics and bacteriophage. Evolutionary Applications. doi:10.1111/j.1752-4571.2012.00248.x

García LV (2004) Escaping the iron claw in ecological studies. Oikos 105:657-663. doi:10.1111/j.0030-1299.2004.13046.x.

Hanski I (1982) On patterns of temporal and spatial variation in animal populations. Ann Zool Fenn 19:21-37

Kilpatrick AM, Ives AR (2003) Species interactions can explain Taylor's power law for ecological time series. Nature 422:65-68. doi:10.1038/nature01471.

Klein AM, Simons BD (2011) Universal patterns of stem cell fate in cycling adult tissues. Development 138:3103-3111. doi:10.1242/dev.060103.

Luria SE, Delbrück M (1943) Mutations of bacteria from virus sensitivity to virus resistance. Genetics 28:491-511

Mathworks (2011) MATLAB R2011b. Mathworks Inc., Natick, Massachusetts, USA

Pal C, Macia MD, Oliver A, Schachar I, Buckling A (2007) Coevolution with viruses drives the evolution of bacterial mutation rates. Nature 450:1079-1081. doi:10.1038/nature06350.

Park H, Cho K (2004) Use of covariates in Taylor's power law for sequential sampling in pest management. J Agric Biol Environ Stat 9:462-478. doi:10.1198/108571104X15746.

Poullain V, Gandon S, Brockhurst MA, Buckling A, Hochberg ME (2008) The evolution of specificity in evolving and coevolving antagonistic interactions between a bacteria and its phage. Evolution 62:1-11

Rainey PB, Bailey MJ (1996) Physical and genetic map of the Pseudomonas fluorescens SBW25 chromosome. Mol Microbiol 19:521-533. doi:10.1046/ j.1365-2958.1996.391926.x.

Ramsayer J, Fellous S, Cohen JE, Hochberg ME (2011) Taylor's law holds in experimental bacterial populations but competition does not influence the slope. Biol Lett. doi:10.1098/rsbl.2011.0895

SAS (2009) JMP statistics and graphics guide (version 8). SAS Institute, Cary, NC, USA

Taylor LR (1961) Aggregation, variance and the mean. Nature 189:732-735. doi:10.1038/189732a0

Taylor RAJ, Woiwod IP, Perry JN (1983) Behavioural dynamics. Nature 303:801-804. doi:10.1038/303801a0

Venail PA, MacLean RC, Bouvier T, Brockhurst MA, Hochberg ME, Mouquet N (2008) Diversity and productivity peak at intermediate dispersal rate in evolving metacommunities. Nature 452:210-214. doi:10.1038/nature06554.

Young $L$, Young JH (1994) Statistics with agricultural pests and environmental impacts. In: Patil GP, Rao CR (ed) Handbook of statistics, vol 12. Environmental Statistics, Elsevier, Amsterdam, pp 735-769

doi:10.1186/2192-1709-1-5

Cite this article as: Kaltz et al: Bacterial microcosms obey Taylor's law: effects of abiotic and biotic stress and genetics on mean and variance of population density. Ecological Processes 2012 1:5. 